\title{
Real-Time Ship Fault Diagnosis Algorithm Based on the Fusion of CNN and RNN
}

\author{
Youyu Zeng ${ }^{+}$, Qiang Xie \\ Nanjing University of Aeronautics and Astronautics, China
}

\begin{abstract}
As the main tool of water transportation, the safety and stability of ship operation play a crucial role in the water transportation industry. However, the existing fault diagnosis methods of ship equipment cannot meet the real-time diagnosis of multiple equipment, to avoid the subjectivity of manual feature extraction and meet the real-time diagnosis of ship faults, designs a real-time fault diagnosis method for ships based on the fusion of convolutional neural network and recurrent neural network in this paper. This method directly inputs the raw data from multiple sensors in a certain window into the network after correlation processing, automatically extract features by convolutional neural network and complete fault diagnosis by recurrent neural network. Experimental results show that this method does not require manual extraction of data features, and its fault diagnosis accuracy is high and the response time is short. Therefore, the method proposed in this paper can be applied to the real time fault diagnosis of ships.
\end{abstract}

Keywords: real-time fault diagnosis of ship faults, convolutional neural network (CNN), recurrent neural network (RNN).

\section{Introduction}

Due to the large number of ship equipment and the complicated operating environment, the probability of equipment failures is greatly increased. If equipment failures cannot be found in time, it will bring great danger to the ship's navigation. Fault diagnosis is of great significance to ship health management through fault detection and fault identification [1]. Effective fault detection can improve the safety of ship, so it is necessary for real-time online fault diagnosis of ship equipment.

Traditional fault diagnosis methods adopt manual feature extraction, relying on signal processing skills and expert experience, which is labor-intensive and time-consuming. The problem of manual feature extraction can be solved by using deep learning method to automatically complete feature learning and fault classification. Yang et al. [2] proposed a fault diagnosis scheme combining hierarchical symbol analysis and convolutional neural network, realized automatic learning of features by using the simplified network structure, and verified its effectiveness in different operating conditions and even different devices. Xu et al. [3] proposed a bearing fault diagnosis method based on multi-level features of wavelet transform, $\mathrm{CNN}$ and $\mathrm{RF}$, and verified that the method has little impact on the classification accuracy of training and test sets with different feature distributions. Jiang et al. [4] proposed a new multi-scale convolutional neural network (MSCNN) structure, which simultaneously carried out multi-scale feature extraction and classification, and verified the superiority of this method for WT gearbox fault diagnosis. S.Udmale et al. [5] proposed a new method for fault classification of rotating machinery based on kurtogram and convolutional neural network, and verified that the method can effectively classify bearing faults under different working conditions. However, due to the numerous sensors that collect the operating status of ship equipment and the time-sequential of the collected data, these methods can not meet the requirements for real-time diagnosis of ship equipment faults.

\footnotetext{
+ Corresponding author. Tel.: + 15651016661;

E-mail address: 1056532596@qq.com
} 
Aiming at the problems of time-consuming and labor-intensive of manual feature extraction, and the timing of signals is not fully utilized in the traditional fault diagnosis using CNN technology, combining with the characteristics of multiple vibration signals generated by a ship at a certain time, a ship fault diagnosis method based on the fusion of CNN and RNN is proposed. The proposed method can avoid the subjectivity of manual feature extraction and meet the real-time diagnosis of ship faults. After comparative experiments, it is found that this method performs well in timeliness and has a high accuracy rate.

\section{Proposed Method}

\subsection{Real-time Diagnosis Process of Ship Fault}

The process of ship fault diagnosis in this paper: Firstly, obtain the raw data collected by multiple sensors with a sliding window. Secondly, perform correlation analysis on the raw data of multiple sensors in the window to enhance the relationship between attributes. Thirdly, use the multi-sensor raw data in the window after correlation analysis as CNN input, and extract features automatically. Fourthly, rearrange the obtained feature sets to obtain the comprehensive feature vectors in the same period. The comprehensive vectors maintain the original relative chronological order, and then take these new vectors as RNN input. Lastly,train the comprehensive feature data using RNN and complete fault classification.

\subsection{Ship Fault Correlation Processing}

There is some correlation between attributes monitored by the ship's multiple sensors. If the raw data of multiple sensors is used directly, the correlation information between attributes will be lost. To overcome this problem, this paper uses the historical data of ships to obtain the correlation matrix between attributes, which can characterize the relationship between attributes. Correlation processing steps are as follows:

- $X=\left(X_{1}, X_{2}, \cdots, X_{m}\right)=\left(x_{i j}\right)_{n \times m}$ represents historical data of multiple sensors in a selected time period, $m$ is the number of sensors, $\mathrm{n}$ is the length of the selected historical data, $x_{i j}$ represents the record value of the $j$-th attribute at the $i$-th time and generally $n>>m$.

- Suppose $X_{i}=\left(x_{1 i}, x_{2 i}, \cdots, x_{n i}\right)^{T}, X_{j}=\left(x_{1 j}, x_{2 j}, \cdots, x_{n j}\right)^{T}$, the correlation between them can be expressed as:

$$
R\left(X_{i}, X_{j}\right)=\frac{\sum_{k=1}^{n}\left(x_{i k}-\bar{x}_{i}\right)\left(x_{j k}-\bar{x}_{j}\right)}{\sqrt{\sum_{k=1}^{n}\left(x_{i k}-\bar{x}_{i}\right)} \sqrt{\sum_{k=1}^{n}\left(x_{j k}-\bar{x}_{j}\right)}}
$$

Where $\bar{x}_{i}$ and $\bar{x}_{j}$ represent the average of attribute $X_{i}$ and attribute $X_{j}$, respectively [6].

- By calculating the correlation matrix $M$ of $X$ is:

$$
M=\left(\begin{array}{cccc}
R\left(X_{1}, X_{1}\right) & R\left(X_{1}, X_{2}\right) & \cdots & R\left(X_{1}, X_{m}\right) \\
R\left(X_{2}, X_{1}\right) & R\left(X_{2}, X_{2}\right) & \cdots & R\left(X_{2}, X_{m}\right) \\
\vdots & \vdots & \ddots & \vdots \\
R\left(X_{m}, X_{1}\right) & R\left(X_{m}, X_{2}\right) & \cdots & R\left(X_{m}, X_{m}\right)
\end{array}\right)
$$

- The ship multi-sensor raw data is divided into time segments of window size, and the number of time segments is calculated according to equation (3).

$$
W_{N}=\frac{S_{L}-W_{L}}{W_{S}}+1
$$

Where $S_{L}$ is the sequence length of the multi-sensor raw data, $W_{L}$ is the length of the sliding window, $W_{S}$ is the step length of the sliding window [7].

- The raw data of multiple sensors in each window is a multivariable time series with a size of $W_{L} \times m$. The multivariate time series in the window is denoted as $Q_{i}\left(i=1,2, \ldots, W_{N}\right), Q_{i}$ at this time can reflect the timing of the raw data of a single sensor, but cannot reflect the correlation between the attributes of multiple sensors. Then, $Q_{i}$ is subjected to correlation processing according to equation (4).

$$
P_{i}=Q_{i} \times M
$$


Where $P_{i}$ represents the multivariate time series after correlation processing, which not only reflects the timeliness of multi-sensor raw data, but also the relevant information between the attributes of the multi-sensors.

\subsection{Ship Fault Feature Extraction}

The raw data collected by the ship's multiple sensors in a certain window after correlation processing is transformed into a multivariate time series $P_{i}$, which is used as an input $X$ of the convolution layer. The size of $P_{i}$ is $W_{L} \times m$ and the number of channels is 1 . The convolutional layer introduces multiple convolution kernels to extract ship fault features. Different convolution kernels extract different fault features [8]. If multiple convolution kernels are combined, a comprehensive ship fault feature extraction can be achieved. Suppose there are $n$ convolution kernels of size $k \times m$, with a step size of $\mathrm{s}$, and a zero-padding operation is performed. Note: The values of $n$ convolution kernels are different, but the width of the convolution kernel is the same as the width of the input $X$. In this way, the input variable $X$ can be transformed into a onedimensional(1-D) feature map after a convolution layer operation. The size of the 1-D feature map is $\left(\frac{W_{L}+\text { Padding }-k}{s}+1\right) \times 1$. The convolution layer convolves the local area of the multivariable time series $X$ in the window with a series of convolution kernels that extract different fault features of the ship to generate a new set of feature maps .A convolution operation of the convolutional layer is shown in Figure 1.

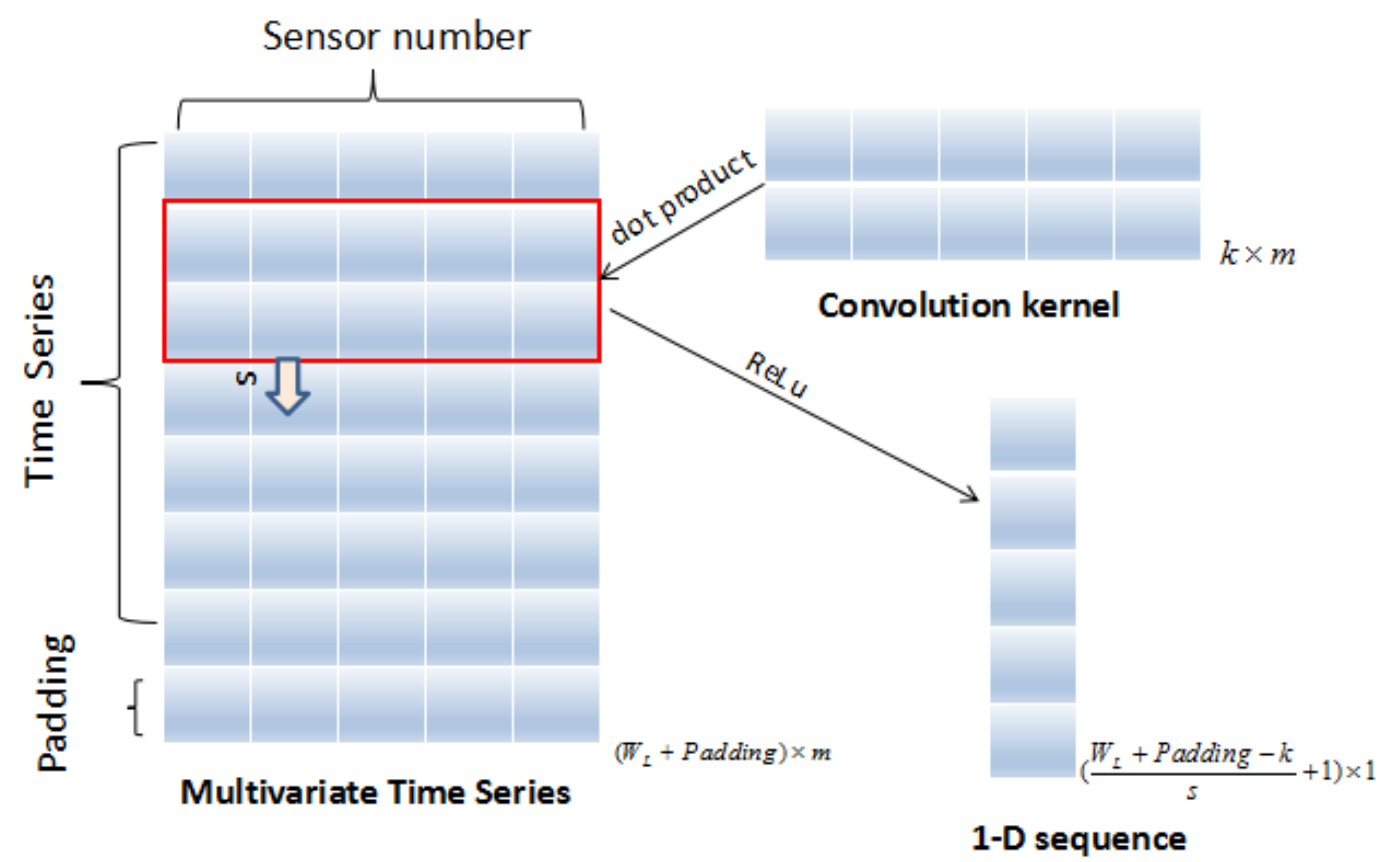

Fig. 1: Convolution operation of a convolution kernel

Different feature maps in Figure 2 represent the results obtained by using different convolution kernels. Boxes of different colors in each feature map represent the results of feature extraction at different time periods. Boxes of the same color of different feature maps represent different results obtained by the convolution operation in the same time period. The learned 1-D feature map containing different fault features of the ship is rearranged to obtain the comprehensive vector of ship fault features in the same time period, and then arrange according to the time sequence of comprehensive vector. The number of comprehensive vectors is equal to the number of 1-D feature maps $\frac{W_{L}+\text { Padding }-k}{s}+1$, and the length of each comprehensive vector is equal to the number of filters $\mathrm{n}$. Each sequence in the Window feature sequence actually corresponds to the raw sequence in the sliding window, maintaining the original relative time order, but just performing a convolution operation in the middle to take these new vectors as the input of RNN. 


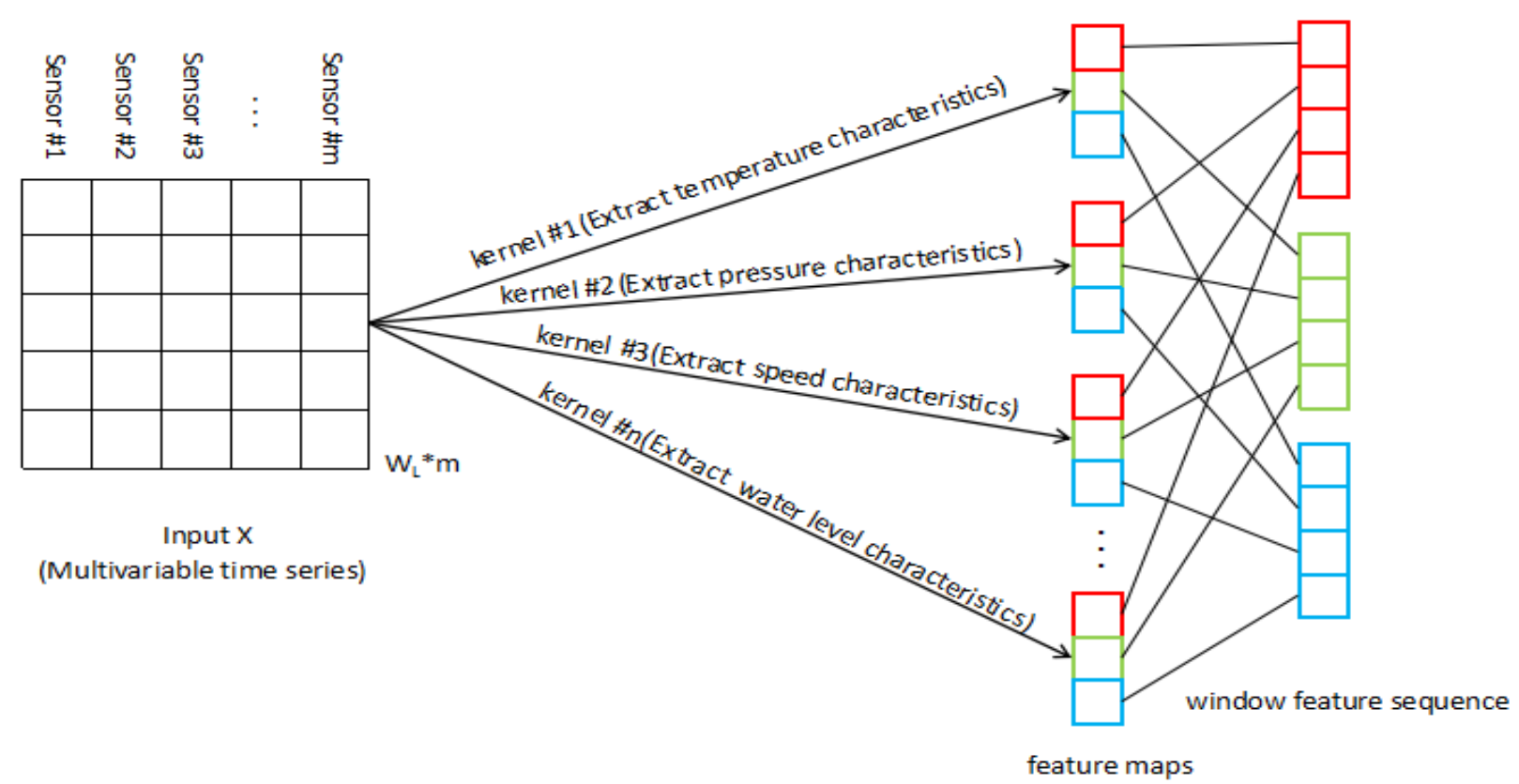

Fig. 2: Network structure of ship fault feature extraction

\subsection{Ship Fault Diagnosis}

The recombined comprehensive feature vector maintains the original relative order and has time sequence. At the same time, the front and rear data of ship equipment are interdependent [9]. In Figure 3, set the red collection as $x_{t-1}$, the green collection as $x_{t}$, and the blue collection as $x_{t+1}$. In the RNN structure, the input at the current moment not only has $x_{t}$ from the input layer, but also a hidden layer state $s_{t-1}$ that provides the previous moment. The RNN processes each comprehensive vector, and stores the current corresponding information in its interior. In the structure proposed in this paper, RNN does not make a decision for each comprehensive feature vector, but processes the information corresponding to all the comprehensive vectors as a whole to classify the entire event. Therefore, RNN proposed in this paper only has an output at the last moment. The output $O=\left(O_{1}, O_{2}, \cdots, O_{r}\right)$ is a probability vector, and $r$ is the number of failure categories (including normal states). $O_{i}(i=1,2, \ldots, r)$ represents the probability that the multivariable time series fault diagnosis result of this window is the fault of type $i$. The fault category is the category corresponding to the component with the largest probability value in $O$.

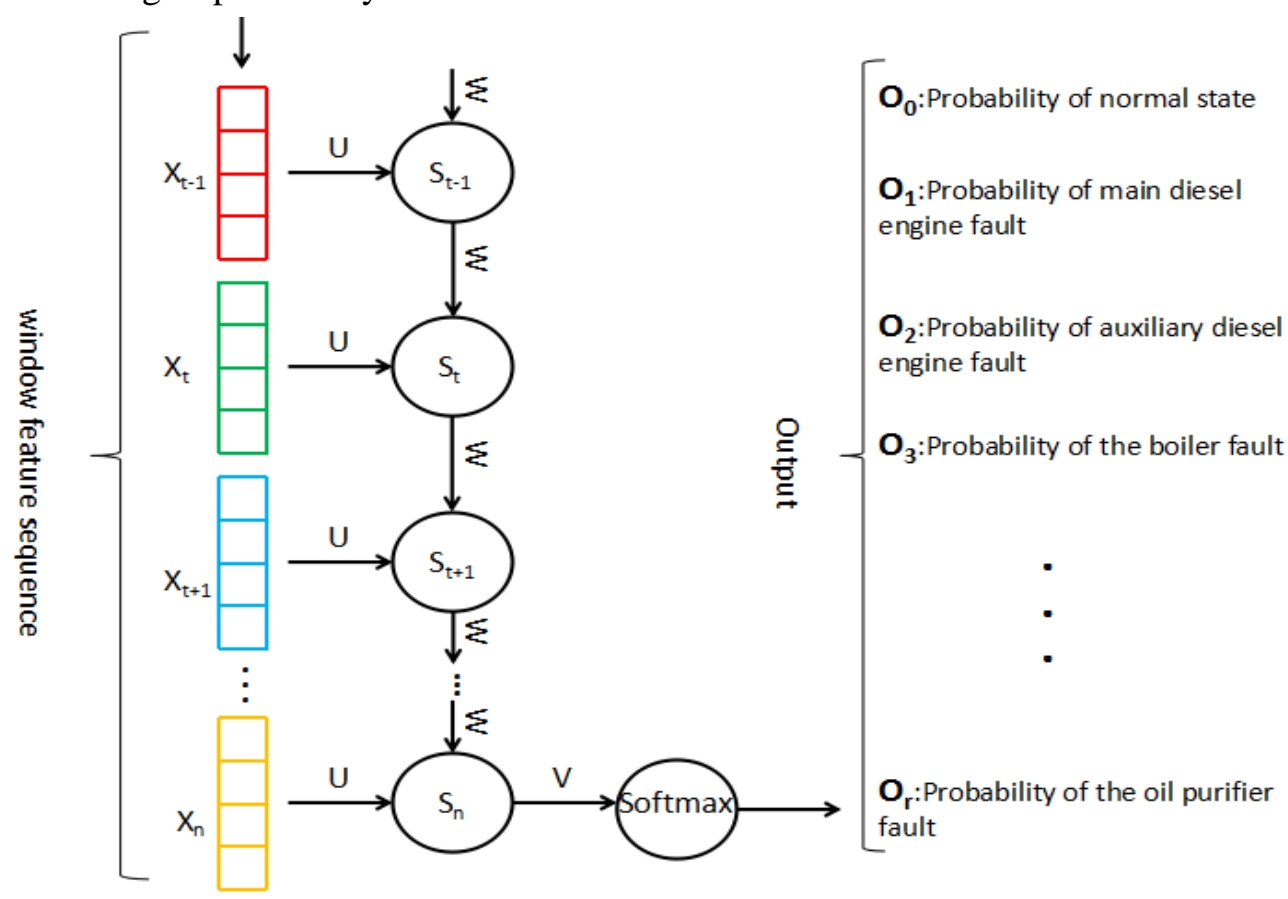

Fig. 3: Network structure of real-time fault diagnosis of ships 


\section{Experiment}

Aiming at the real-time operating status of a ship's equipment, the normal operation of the system and seven other fault conditions (main diesel engine failure, auxiliary diesel engine failure, boiler failure, air compressor failure, hot oil heater failure, oil purifier failure, and exhaust gas boiler failure) were selected. In order to facilitate computer processing, we use $0,1,2,3,4,5,6$, and 7 to represent the eight operating states of the ship system. The number 0 represents the normal state, and so on. A sliding window with a length of 80 is used and the sliding distance of the window is set to 1 to meet the real-time nature of ship fault diagnosis. Each failure state collection contains 4000 samples, of which 3000 samples are used as training data and 1000 samples are used as test data.

The method proposed in this paper converges fast on the training set. After 10 rounds of iterations, the training accuracy is about $100 \%$ and the loss is about 0.001 . As can be seen from Figure 4 , in the test set, the fault diagnosis accuracy of ships in normal state is $99.4 \%$, while the fault diagnosis accuracy of main and auxiliary diesel engines is slightly lower, because there is a strong connection between them, and the fault diagnosis effect of other states is also better. According to Table 1, the SVM fault diagnosis scheme has great advantages in time, but the accuracy rate is low. The accuracy rate of fault diagnosis of ship equipment using RNN alone is lower than that of using CNN alone, because RNN cannot automatically extract fault features and needs to be manually extracted. The quality of the extracted features affects the accuracy of diagnosis. However, because the data collected by the ship is time-series, the advantages of RNN processing time-series data are reflected in the time response. The diagnostic method proposed by S.Udmale and Zhang has high accuracy, but it also has a long response time, so it is not suitable for real-time fault diagnosis of ships. The fault diagnosis method proposed in this paper is slightly lower in accuracy than the fault method proposed by S.Udmale, but the response time is much shorter than the fault method proposed by S.Udmale, which is the shortest response time among the compared methods. The ship fault diagnosis method proposed in this paper has a test accuracy rate of up to $98.41 \%$, and the average response time of each test sample is about $16.4 \mathrm{~ms}$, which meets the requirements of real-time online fault diagnosis.

In this paper, the multi-sensor raw data in the window is correlation processed firstly and then directly used as the input of the network to automatically extract the fault features without manual intervention. The difficulty of network training is low and the diagnosis effect is good, and it has achieved good results in ship fault diagnosis experiments. The response time has obvious advantages over several other fault diagnosis methods. The test results of each sample only take about $16.4 \mathrm{~ms}$, which can meet the real-time online diagnosis requirements and guide the fault maintenance strategy of a ship in the working state in time.

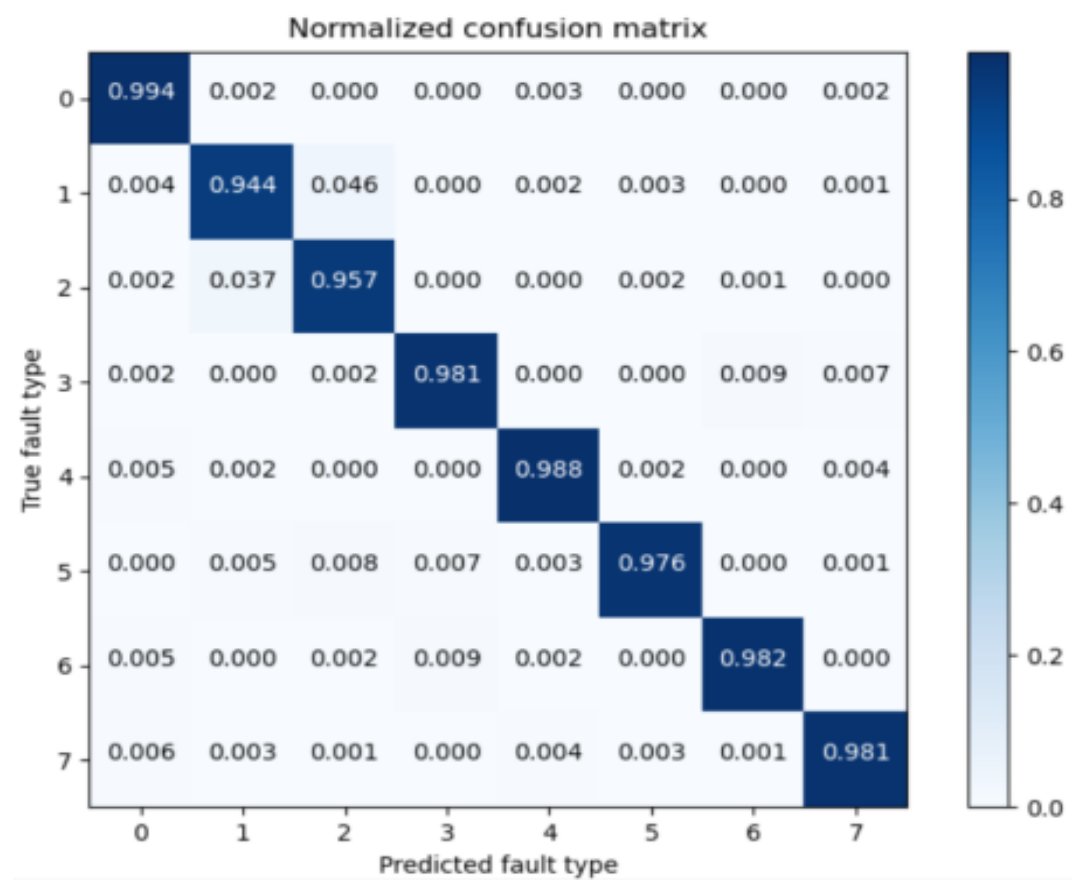

Fig. 4: The confusion matrix on the test set 
Table 1: Comparison of the diagnostic results of the test set using different methods

\begin{tabular}{ccc}
\hline Methods & Accuracy(\%) & Average Times(ms) \\
\hline SVM & 91.22 & 20.6 \\
\hline RNN & 94.57 & 26.2 \\
\hline CNN & 96.81 & 34.1 \\
\hline S.Udmale Proposed & 98.67 & 34.6 \\
\hline Zhang Proposed & 98.23 & 34.8 \\
\hline Proposed method & 98.41 & 16.4 \\
\hline
\end{tabular}

\section{Conclusion}

In this paper, aiming at the demand of real-time fault diagnosis of ship, combined with the advantages of $\mathrm{CNN}$ and RNN, the fusion technology of CNN and RNN is proposed for fault diagnosis of ships. This method closely correlates the ship's multi-feature parameters through correlation processing, then uses CNN to automatically extract ship fault features, next uses RNN to find temporal features, and finally performs fault classification. It is verified by experiments that the diagnostic model proposed in this paper has a good effect in the classification of ship fault diagnosis, and the time response is fast, which can meet the needs of real-time ship fault diagnosis.

On the other hand, there is a certain coupling relationship between some ship fault instances. So, if a failure of the ship is found, other related equipment should be tested. Therefore, the further work can be to use the association rule mining algorithm to avoid secondary diagnosis and localization of the fault and save a lot of fault diagnosis costs.

\section{References}

[1] I. Lazakis, Y. Raptodimos,T. Varelas. Predicting ship machinery system condition through analytical reliability tools and artificial neural networks[J]. Ocean Engineering,2018,152.

[2] Yang Yuantao,Zheng Huailiang,et al. A fault diagnosis scheme for rotating machinery using hierarchical symbolic analysis and convolutional neural network. [J]. ISA transactions,2019,91.

[3] Xu Gaowei,Liu Min,et al. Bearing Fault Diagnosis Method Based on Deep Convolutional Neural Network and Random Forest Ensemble Learning[J].Sensors,2019,19(5):1088.

[4] Guoqian Jiang,Haibo H,et al. Multiscale Convolutional Neural Networks for Fault Diagnosis of Wind Turbine Gearbox[[J].IEEE TRANSACTIONS ON INDUSTRIAL ELECTRONICS.2019,66(4):3196-3207.

[5] Sandeep S.Udmale,Sangram S.Patil,et al.A bearing vibration data analysis based on spectral kurtosis and ConvNet[J].Soft Computing.2019,23:9341-9359.

[6] Zhang kunhua,Ding lixin,Wan runze. Multivariate time series attribute selection based on correlation density [J]. Computer application and software, 2017,34 (12): 273-277 + 320 .

[7] Mikel Canizo,Isaac Triguero,Angel Conde,Enrique Onieva. Multi-head CNN-RNN for multi-time series anomaly detection: An industrial case study[J]. Neurocomputing,2019,363.

[8] Jiangquan ZHANG, Yi SUN, et al. A new bearing fault diagnosis method based on modified convolutional neural networks[J]. Chinese Journal of Aeronautics,2020,33(02):439-447.

[9] Samuel Würtz,Ulrich Göhner. Driving Style Analysis Using Recurrent Neural Networks with LSTM Cells[J]. Journal of Advances in Information Technology,2020,11(01):1-9. 